\title{
MANAGEMENT OF COMMERCIAL CREDIT GRANTED AND EARNED AND ITS ROLE IN MARKET VALUEAPPLIED RESEARCH FOR A SAMPLE OF COMPANIES LISTED ON THE IRAQ STOCK EXCHANGE
}

\author{
*Prof. Dr. Abdul Salam Said gesture, **Ibraheemabdulh \\ *University of Baghdad - College of Management and Economics - Department of Business Administration \\ **College of Imam Kadhim (AS) Department of Business Administration
}

DOI: $10.37648 /$ ijrssh.v10i01.011

Received:01 ${ }^{\text {st }}$ October, 2019; Accepted:30th October, 2019; Published: $20^{\text {th }}$ November, 2019

\begin{abstract}
This research comes in the context of knowing the dialectic of the relationship between commercial credit granted and earned and its impact on the market value of companies, which gives the management of companies the freedom to make the appropriate financial decision in the aspects of finance (short-term) and investment (short-term) The above three research for (33) companies listed in the Iraqi market for securities and four sectors (industrial, agricultural, services, tourism and hotels) and for five years from (2013-2017) based on a number of statistical methods are recognized and innovative research service, has Policies were discussed Credit approved by companies (risk, moderate, conservative) for the credit granted and earned to access the nine alternatives (conservative - conservative, moderate - conservative, risk - conservative, conservative - moderate, moderate - moderate, risk - moderate conservative, risk - moderate And stand on the level of efficiency of the departments of those companies (high - high, moderate - high, low - high, high moderate, moderate - moderate, low - moderate, high - low, moderate - low, low - low. The research concluded that the statistical tests among the variables of the study proved that the companies that recorded the highest market value of the company are obtaining a credit earned greater than the credit granted, i.e. the moderate policy of credit granted and the risk policy of credit earned, it indicates that the market value is high due to the average efficiency of the grantee. In addition, high efficiency of the gainer. The researcher recommends the necessity of adopting such variables in the studies of companies and generalizing their results to the society and not neglecting them to know the most important conclusions reached in order to give a clear vision on the importance of studying these variables and the necessary ways to find appropriate management for them at the level of commercial credit granted and their reflection on other objectives. The highest is to maximize shareholder value )
\end{abstract}

Keywords: Commercial Credit credit, credit granted, credit earned, company value 


\section{INTRODUCTION}

The management of commercial credit granted and acquired is not a new phenomenon in business companies, since the commercial credit granted and earned existed in the past the presence of organizations, which constitutes a very large proportion of the balance sheet and income statement, and commercial credit granted and earned is an important element of the activities of business companies, where credit Granted form a very large proportion compared to current assets, as is the case of credit earned where the largest proportion compared to short-term liabilities, as commercial credit is the beginning and end of the working capital cycle, and it maintains continuity Business Moreover, the right policy of commercial credit is granted and earned in all business operations based on commercial solvency. The lack of funds, the high cost and the difficulty of lending at present have created a serious problem for business companies, it must be the presence of commercial credit like any other assets of the company and deal with it as an investment vehicle through the granted credit and financing from the direction of credit earned, moreover today the focus is on providing The optimum volume of trade credit at the right time and at the right cost. Trade credit consists of two different types: grantee (also referred to as accounts receivable, short-term investment, accounts receivable, accounts receivable) which is on the asset side of the budget. A short-term investment, acquired (also referred to as accounts payable, short-term financing, management of creditors, accounts payable) which is on the liabilities side of the balance sheet and is a short-term financing component.

Commercial credit management has become more important in the modern world in business due to the change in business management, increasing difficulties, the cost of borrowing and technological development, and the appropriate policy of granted and earned credit is carried out through a set of policies (conservative, moderate, risky) and all these practices. These policies contribute to the achievement of the company's objectives of maximizing market value as well as the above-mentioned practices.

\section{THEORETICAL FRAMEWORK:}

\section{2-1 Management of commercial credit granted}

Commercial credit granted is an implicit contract where suppliers deliver goods to customers and do not pay in cash, but promise to pay later. This promise represents a credit or an implicit contract. ( $\mathrm{Wu}, \&$ et... al, 2014: 146)) Commercial credit is the emerging credit between the company and the buyer through forward sales (Shamaa, 283: 1992). Credit management is the decision-making process of investing in granted credit, where Credit is necessary to increase sales and profits of the company the goal of credit management Man granted is to make a sound decision with regard to investing in credit and promoting sales and profits to that point where the return on investment in additional financing of receivables is lower than the cost of funds raised to finance this additional credit (Road Ehrhardt\& Brigham, 2012). There are a number of determinants that affect the granting of commercial credit (Chou \& et... al, 2011: 279-280): -

A. Sales: The company may extend commercial credit more aggressively to promote sales, leading to a positive relationship between sales and accounts receivable, there will be a correlation between sales and accounts receivable.

B. Cash (cash register): If companies with more liquidity relative to assets will have more cash and be able to provide more trade credit, as this will be a positive relationship between cash and accounts receivable, this variable has a positive coefficient. .

C. Inventory: For inventory-based management purposes, companies with more stocks are likely to offer more business credit than other companies. Both inventories and accounts receivable are current assets and are therefore alternatives from the perspective of asset management.

D. . Company size $(\log \mathrm{A})$ : The impact of company size is the agent of market energy, from financial assistance Opinion suggests that large companies offer more business credit. In contrast, quality assurance theory suggests that small businesses need to ensure the quality of 
their products more than larger and more established companies and thus offer commercial credit.

\section{2-2- Management of Earned Commercial Credit}

Credit earned is the flow of goods or services from abroad into the company. Credit earned can also be defined as "obtaining goods and services in return for a promise to pay in cash at a specified time in the future. Credit is based on trust and trust from the acquiring party and requires a period of time between the time of receipt or lending and payment. Ehrhardt\& Brigham, 2012: Earned credit is a financing tool if the use of earned credit gives a better chance of accessing external capital or reducing the cost of capital, it gives the buyer a comparative advantage in the product market 2012: 50), Olusola\&Olusola (2006: 1200). Pike et al., 2939 (2007), Beck, encourages SMEs to For earned credit and other short-term financing because these financial sources often do not involve any application for collateral, Öhman (2016: 3) that earned credit is important for companies with difficulty borrowing from financial markets where alternative sources are Finance is mostly unavailable or when the financial sector is weak. Moreover, earned credit is more important for unlisted companies because they are restricted from access to capital markets (Dohaiman, 2013: 206). Not less credit can also be used Because they can go to other sources of finance due to their credit capacity and reputation, earned credit is even more important when companies are smaller, younger and more obscure, (Niskanen\&Niskanen, 2006: 92) that internal finance may also affect the company's liquidity position. On demand for credit earned (FISMAN \& LOVE, 2003: 353 ), and thus will reduce their demand for financing through suppliers (Teruel\& Solano, 2008: 10), and it often happens that companies face difficulties in accessing financial markets and that Financing buys on credit, which means using earned credit Kwenda, 2014: 433)), works credit Taman Acquisition mainly as an alternative to bank borrowing (2016: 139, Mişu\&Deari) Empirical evidence suggests that for most non-financial companies during the credit earned is an important source of financing and credit earned represents about $41 \%$ and $35 \%$ of total debt among medium-sized companies in the states USA and USA respectively represent more than $50 \%$ of short-term debt in both countries (Teruel\& Solano, 2010: 448).

\section{2-3 Market value of the company}

The market value of the company's shares is calculated by multiplying the market price of the common stock by the number of shares issued (Amri, 2010: 426), and companies are looking to maximize their profits. The Company's value is obtained by discounting the expected cash flows, and the value of the companies is the present value of the expected cash flows from all existing investments and potential future growth, discounted from the cost of capital 76 (Damadoran, 2002). Determine s The value of any asset is usually done by market forces, and similarly in the case of stock prices, they appear in circulation among investors in the stock markets. Inflation puts investors in the stock market to earn a return on their investment and the shares are sold from one investor to another. This transaction depends on the level of information owned by each participant. , An investor that improves the company's performance prediction may want to buy that company's shares, and in this way depends on the level of information to a certain extent in determining the share price (Malik et al, 2012).

The researcher also defines the market value of companies as they anticipate what will be the value of shares in financial markets in which are directly related to the size of real investments and future profits.

\section{3- METHODS AND TOOLS:}

\section{3-1 Objective of the research}

Measure the success of financial management in companies Sample research in the management of commercial credit granted in order to raise the levels of market value by determining the credit policy adopted and the efficiency of credit management, and measure the success of financial management in companies Sample research in the management of commercial credit earned leading to raise Levels of market value through determining the credit policy adopted and the efficiency of credit management, and trying to reach the determination of the best policy or the best level of credit granted, the best policy and the size of the best financing through the earned credit, and try to To 
ascertain the likelihood of one or more of the granted and earned commercial credit policies (conservative, moderate, and risky) adopted by the research to explain the relationship between the management of granted and earned credit and the market value of the company and to show the strength of that relationship on the market value of the company.

\section{3-2 Research Tools and Model:}

1. Credit policy measures:

A. Credit granted turnover $=$ net sales $/$ credit granted (Dary\& James, 2018: 43), (Amri, 2007: 128) ..1

B. Average collection period $=360$ days $/$ total credit granted. (Happy, 2019)........2

C. Current Ratio $=$ Current Assets / Current Liabilities (Amri, 2007) .......... 3

D. Inventory turnover $=$ sales $/$ inventory. (Amiri, 2007: 128) 4

E. Provision for doubtful debts $=$ part $/$ all 5

2. Credits of earned credit policy:
A. Credit earned to total short-term liabilities = credit earned / total short-term liabilities (NTHENGE, 2013: 11) 11

B. Credit earned to total liabilities

C. Credit earned to total liabilities $=$ credit earned / total liabilities. Dary\& James, 2018: 43)) 10.

D. Credit earned to total short-term liabilities = credit earned / total liabilities and equity .. 11

E. Credit Earned $=$ Part $/$ All 12

\section{Earned Credit Efficiency Measures:}

A. Average repayment period $=360$ days $/$ credit earned turnover (Said, 2019: 220) ............. 13

B. Credit Earned Turnover $=$ Purchases $/$ Earned Credit Turnover. (Happy, 2019: 220) 14

Figure (1) illustrates the research model and the nature of the relationship between the granted and acquired credit policies as well as their efficiency.

Table (1) illustrates the policy mix of commercial credit granted and earned to the research sample companies listed on the Iraq Stock Exchange.

Table (1) Type of commercial credit policies granted and earned

\begin{tabular}{|c|c|c|c|}
\hline Conservative politics & Moderate policy & Risky politics & Credit granted \\
\hline Risky, conservative & Risky, moderate & Risky, risky & Risky politics \\
\hline Moderate, conservative & Moderate, moderate & Moderate, risky & Moderate polic \\
\hline Conservative & Conservative, moderate & Conservative, risk & Conservative politics \\
\hline
\end{tabular}




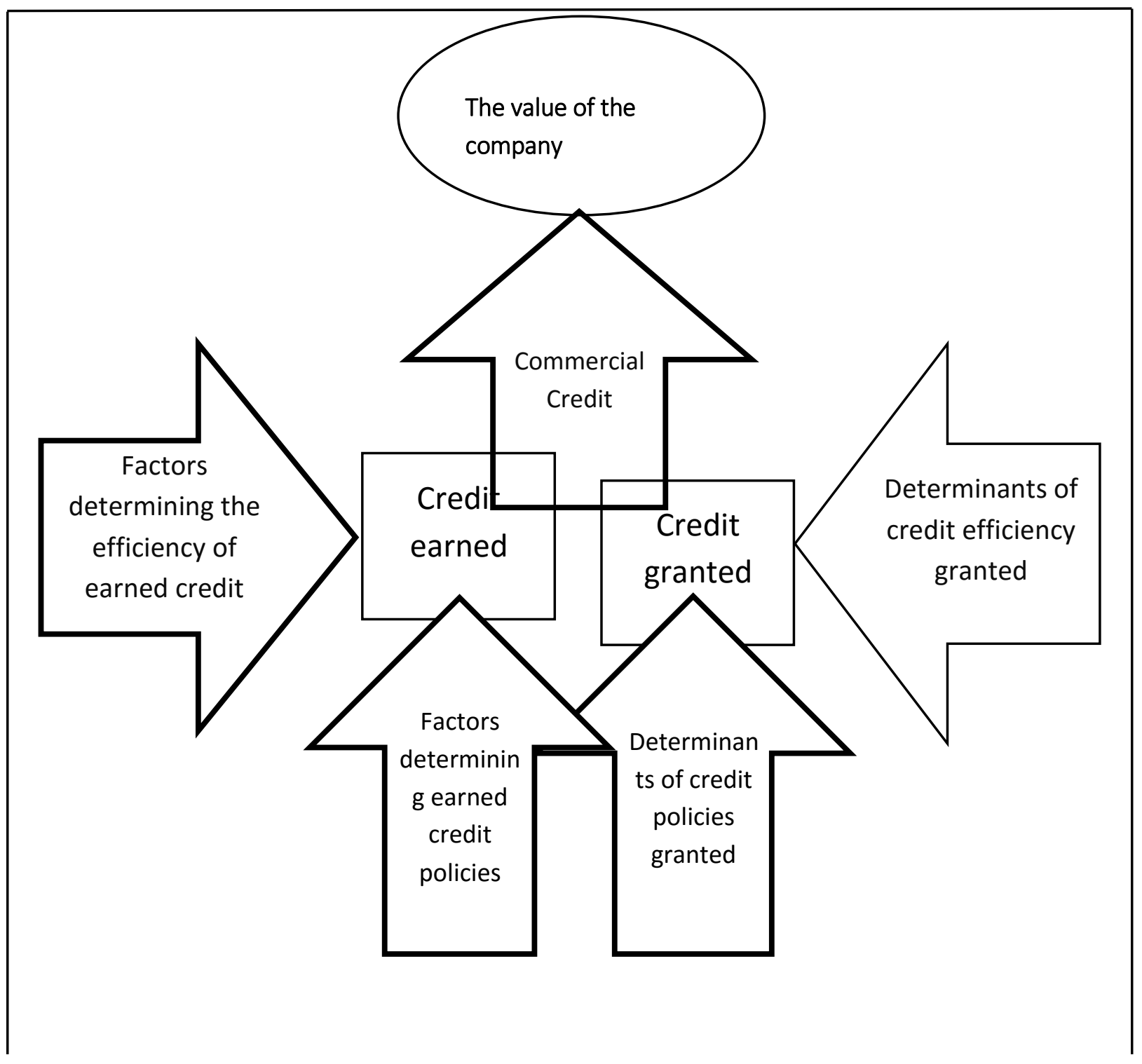

Figure (1) Research Model

\section{PRACTICAL FRAMEWORK OF RESEARCH:}

\section{4-1 Granted commercial credit policies}

It was found that the cumulative grades ranged between (0.01) minimum as in the Iraqi company for carpets and furnishings in 2014 and in Karbala hotels in 2015, and (0.69) degree as a maximum in the Baghdad Public Transport Company, where the percentages were extracted Through the measures of credit policies granted in Table (2) based on the credit measures granted in the above paragraph. 
Table (2) Cumulative Credit Scores for Companies Sample Research

\begin{tabular}{|c|c|c|c|c|c|c|}
\hline 2017 & 2016 & 2015 & 2014 & 2013 & The Company's name & $ت$ \\
\hline 0.25 & 0.24 & 0.28 & 0.14 & 0.30 & National Chemical Industries & 1 \\
\hline 0.18 & 0.17 & $\mathbf{0 . 0 3}$ & 0.01 & 0.05 & Iraqi Carpets \& Furniture & 2 \\
\hline 0.24 & 0.12 & 0.17 & 0.10 & 0.22 & $\begin{array}{l}\text { Baghdad Packing Materials } \\
\text { Industry }\end{array}$ & 3 \\
\hline 0.11 & 0.08 & 0.04 & 0.12 & 0.25 & Baghdad Soft Drinks & 4 \\
\hline 0.29 & 0.33 & 0.52 & 0.41 & 0.36 & $\begin{array}{l}\text { Readymade Garments Production } \\
\text { \& General Trading }\end{array}$ & 5 \\
\hline 0.31 & 0.32 & 0.32 & 0.40 & 0.29 & Alhlal Industrial & 6 \\
\hline 0.28 & 0.41 & 0.26 & 0.18 & 0.14 & Modern sewing & 7 \\
\hline 0.19 & 0.28 & 0.03 & 0.02 & 0.24 & Modern chemical products & 8 \\
\hline 0.29 & 0.10 & 0.12 & 0.20 & 0.20 & $\begin{array}{l}\text { Canadian Vaccines and } \\
\text { Veterinary Medicines }\end{array}$ & 9 \\
\hline 0.16 & 0.41 & 0.39 & 0.32 & 0.47 & $\begin{array}{l}\text { AL MANSOUR MEDICAL \& } \\
\text { BEAUTY INDUSTRIES }\end{array}$ & 10 \\
\hline 0.19 & 0.13 & 0.20 & 0.08 & 0.24 & Dyes industry & 11 \\
\hline 0.16 & 0.22 & 0.37 & 0.15 & 0.29 & Alhlal Industrial & 12 \\
\hline 0.54 & 0.58 & 0.57 & 0.63 & 0.49 & Sudair hotels & 13 \\
\hline 0.44 & 0.37 & 0.26 & 0.50 & 0.48 & AL MANSOUR hotels & 14 \\
\hline 0.06 & $\mathbf{0 . 0 3}$ & 0.01 & 0.15 & 0.06 & Karbala hotels & 15 \\
\hline 0.64 & $\mathbf{0 . 4 7}$ & 0.67 & 0.54 & 0.33 & The tourist city of Mosul Dam & 16 \\
\hline 0.29 & 0.20 & 0.43 & 0.42 & 0.20 & Babylon Hotel & 17 \\
\hline 0.20 & 0.19 & 0.13 & 0.17 & $\mathbf{0 . 1 3}$ & Baghdad Hotel & 18 \\
\hline 0.44 & 0.35 & 0.26 & 0.22 & 0.18 & Palestine Hotel & 19 \\
\hline 0.17 & 0.15 & 0.17 & 0.10 & 0.31 & National Tourism Investment & 20 \\
\hline 0.43 & 0.52 & 0.42 & 0.44 & 0.23 & ISHTAR hotels & 21 \\
\hline 0.48 & 0.18 & 0.28 & 0.21 & 0.04 & $\begin{array}{l}\text { Iraqi Agricultural Production and } \\
\text { Marketing }\end{array}$ & 22 \\
\hline 0.56 & 0.24 & 0.12 & 0.15 & 0.28 & National Agricultural Production & 23 \\
\hline 0.55 & 0.29 & 0.57 & 0.30 & $\mathbf{0 . 5 3}$ & $\begin{array}{l}\text { Iraqi Dates Manufacturing \& } \\
\text { Marketing }\end{array}$ & 24 \\
\hline 0.18 & 0.18 & 0.24 & 0.11 & 0.16 & $\begin{array}{l}\text { Middle East Lotus Fish } \\
\text { Production }\end{array}$ & 25 \\
\hline 0.62 & 0.48 & 0.59 & 0.52 & 0.55 & $\begin{array}{l}\text { Iraqi meat production and } \\
\text { marketing }\end{array}$ & 26 \\
\hline 0.42 & 0.42 & 0.42 & 0.42 & 0.42 & $\begin{array}{l}\text { Modern Animal and Agricultural } \\
\text { Production }\end{array}$ & 27 \\
\hline 0.20 & 0.25 & 0.20 & 0.15 & 0.23 & Saliva Karkh tourist city & 28 \\
\hline 0.40 & 0.25 & 0.38 & 0.25 & 0.27 & Mosul City Games & 29 \\
\hline 0.46 & 0.28 & 0.26 & 0.31 & 0.46 & Alnkba General Contracting & 30 \\
\hline 0.32 & 0.30 & 0.27 & 0.27 & 0.39 & $\begin{array}{l}\text { Maamoura Real Estate } \\
\text { Investments }\end{array}$ & 31 \\
\hline 0.34 & 0.19 & 0.34 & $\mathbf{0 . 3 3}$ & 0.34 & Al Ameen Real Estate Investments & 32 \\
\hline
\end{tabular}




\begin{tabular}{|l|l|l|l|l|l|l|}
\hline 0.29 & 0.28 & 0.58 & 0.69 & 0.30 & $\begin{array}{l}\text { Baghdad General Transport \& } \\
\text { Real Estate }\end{array}$ & 33 \\
\hline
\end{tabular}

The policy is calculated by the following steps

1 - Relying on the upper and lower limits to determine the range of each research sample companies and divide it into three categories to determine the length of the approved category.

2 - Determine the upper and lower limits for each of the three categories, to represent the first category conservative policy and the second policy of moderate and third policy of risk.

Through the above, the commercial credit policies granted to the business sample companies for the years (2013-2017), as shown in Table (3), will be determined by the following equations:

Range $=$ High - Low $=0.69-0.01=0.68$

The length of the class $=0.68 \div 3=0.22,6$

Minimum reserve policy $=0.01$

Maximum conservative policy $=0.01+0.22,6=0.23,6$

Minimum moderate policy $=0.23 .7$

The upper limit of moderate policy $=0.23 .7+0.22 .6=0.46 .3$

Minimum risk policy $=0.46 .4$

Maximum risk policy $=0.46 .4+0.22 .6=0.69$

Table (3) Limits of Corporate Policies Research Sample

\begin{tabular}{|c||c||l||}
\hline maximum & minimum & Policy type \\
\hline \hline $\mathbf{0 . 2 3 , 6}$ & 0.01 & Conservative politics \\
\hline \hline $\mathbf{0 . 4 6 , 3}$ & $0.23,7$ & Moderate policy \\
\hline \hline $\mathbf{0 . 6 9}$ & $0.46,4$ & Risky politics \\
\hline \hline
\end{tabular}

\section{4-2 Determination of Earned Credit Policy}

Table (4) shows the measurement of commercial credit earned

Table (4) Cumulative Analysis of Credit Acquired for Years and Sample Companies

\begin{tabular}{|c|c|c|c|c|c|c||}
\hline \hline 2017 & 2016 & 2015 & 2014 & 2013 & The Company's name & $\Xi$ \\
\hline \hline 0.28 & 0.57 & 0.53 & 0.57 & 0.56 & $\begin{array}{l}\text { National Chemical } \\
\text { Industries }\end{array}$ & 1 \\
\hline
\end{tabular}




\begin{tabular}{|c|c|c|c|c|c|c|}
\hline 0.41 & 0.54 & 0.57 & 0.54 & 0.61 & $\begin{array}{l}\text { Iraqi Carpets \& } \\
\text { Furniture }\end{array}$ & 2 \\
\hline 0.51 & 0.51 & 0.50 & 0.51 & 0.50 & $\begin{array}{l}\text { Baghdad Packing } \\
\text { Materials Industry }\end{array}$ & 3 \\
\hline 0.35 & 0.38 & 0.53 & $\mathbf{0 . 3 3}$ & 0.40 & Baghdad Soft Drinks & 4 \\
\hline 0.41 & 0.59 & 0.59 & 0.59 & 0.63 & $\begin{array}{l}\text { Readymade Garments } \\
\text { Production \& General } \\
\text { Trading }\end{array}$ & 5 \\
\hline 0.56 & 0.67 & 0.56 & 0.67 & 0.55 & Alhlal Industrial & 6 \\
\hline 0.37 & 0.50 & 0.44 & 0.53 & 0.51 & Modern sewing & 7 \\
\hline 0.52 & 0.58 & 0.62 & 0.58 & 0.35 & $\begin{array}{l}\text { Modern chemical } \\
\text { products }\end{array}$ & 8 \\
\hline 0.29 & 0.29 & 0.41 & 0.51 & 0.29 & $\begin{array}{l}\text { Canadian Vaccines and } \\
\text { Veterinary Medicines }\end{array}$ & 9 \\
\hline $\mathbf{0 . 6 0}$ & 0.56 & 0.54 & 0.62 & 0.52 & $\begin{array}{l}\text { AL MANSOUR } \\
\text { MEDICAL \& BEAUTY } \\
\text { INDUSTRIES }\end{array}$ & 10 \\
\hline 0.28 & 0.29 & 0.27 & 0.53 & 0.27 & Dyes industry & 11 \\
\hline 0.53 & 0.52 & 0.53 & $\mathbf{0 . 5 3}$ & 0.53 & Alhlal Industrial & 12 \\
\hline 0.67 & 0.52 & 0.58 & 0.52 & 0.56 & Sudair hotels & 13 \\
\hline 0.59 & 0.70 & 0.62 & 0.70 & 0.60 & AL MANSOUR hotels & 14 \\
\hline 0.45 & 0.35 & 0.48 & 0.54 & 0.30 & Karbala hotels & 15 \\
\hline 0.52 & 0.59 & 0.63 & 0.59 & 0.61 & $\begin{array}{l}\text { The tourist city of Mosul } \\
\text { Dam }\end{array}$ & 16 \\
\hline 0.62 & 0.72 & 0.62 & 0.72 & 0.74 & Babylon Hotel & 17 \\
\hline 0.55 & 0.52 & 0.55 & 0.58 & 0.49 & Baghdad Hotel & 18 \\
\hline 0.62 & 0.60 & 0.62 & 0.61 & 0.64 & Palestine Hotel & 19 \\
\hline 0.51 & 0.51 & 0.51 & 0.51 & 0.52 & $\begin{array}{l}\text { National Tourism } \\
\text { Investment }\end{array}$ & 20 \\
\hline 0.68 & 0.16 & 0.69 & 0.61 & 0.18 & ISHTAR hotels & 21 \\
\hline 0.52 & 0.52 & 0.53 & 0.53 & 0.53 & $\begin{array}{l}\text { Iraqi Agricultural } \\
\text { Production and } \\
\text { Marketing }\end{array}$ & 22 \\
\hline 0.54 & 0.57 & 0.53 & 0.57 & 0.57 & $\begin{array}{l}\text { National Agricultural } \\
\text { Production }\end{array}$ & 23 \\
\hline 0.63 & 0.54 & 0.54 & 0.53 & 0.53 & $\begin{array}{l}\text { Iraqi Dates } \\
\text { Manufacturing \& }\end{array}$ & 24 \\
\hline
\end{tabular}




\begin{tabular}{|c|c|c|c|c||l|l|}
\hline 0.54 & 0.52 & 0.52 & 0.56 & 0.51 & $\begin{array}{l}\text { Marketing } \\
\text { Production }\end{array}$ & 25 \\
\hline 0.58 & 0.57 & 0.42 & 0.57 & 0.57 & $\begin{array}{l}\text { Iraqi meat production } \\
\text { and marketing }\end{array}$ & 26 \\
\hline 0.56 & 0.52 & 0.47 & 0.51 & 0.52 & $\begin{array}{l}\text { Modern Animal and } \\
\text { Agricultural Production }\end{array}$ & 27 \\
\hline 0.44 & 0.35 & 0.28 & 0.58 & 0.28 & Saliva Karkh tourist city & 28 \\
\hline 0.57 & 0.57 & 0.57 & 0.57 & 0.57 & Mosul City Games & 29 \\
\hline 0.18 & 0.22 & 0.19 & 0.51 & 0.22 & $\begin{array}{l}\text { Alnkba General } \\
\text { Contracting }\end{array}$ & 30 \\
\hline 0.52 & 0.52 & 0.52 & 0.53 & 0.55 & $\begin{array}{l}\text { Maamoura Real Estate } \\
\text { Investments }\end{array}$ & 31 \\
\hline 0.62 & 0.50 & 0.62 & 0.50 & 0.50 & $\begin{array}{l}\text { Al Ameen Real Estate } \\
\text { Investments }\end{array}$ & 32 \\
\hline 0.50 & 0.31 & 0.50 & 0.51 & 0.31 & $\begin{array}{l}\text { Baghdad General } \\
\text { Transport \& Real Estate }\end{array}$ & 33 \\
\hline
\end{tabular}

The policy is calculated according to the steps adopted in Table (3) to extract the earned credit policies as in Table (5).

Table (5) Limits of Corporate Policies Research Sample

\begin{tabular}{|c|c|l|}
\hline maximum & minimum & Policy type \\
\hline $0.35,3$ & 0.16 & Conservative politics \\
\hline $0.54,7$ & $0.35,4$ & Moderate policy \\
\hline $0.74,1$ & $0.54,8$ & Risky politics \\
\hline
\end{tabular}

4-3 - Analyze and market value levels: - Analyze and market value levels

Table (6) shows the analysis of companies listed on the Iraqi Stock Exchange for the years 2013-2017) and for four sectors, namely the industrial sector, the hotel sector, tourism, agriculture and services.

Table (6) Market Value of Companies Sample Research Note that the figures

\begin{tabular}{|c|c|c|c|c|c|c|c|c|c|c|c|}
\hline $\begin{array}{c}\text { Coefficient } \\
\text { of } \\
\text { variation }\end{array}$ & $\begin{array}{c}\text { standard } \\
\text { deviation }\end{array}$ & $\begin{array}{c}\text { the } \\
\text { average }\end{array}$ & minimum & maximum & 2017 & 2016 & 2015 & 2014 & 2013 & $\begin{array}{l}\text { The Company's } \\
\text { name }\end{array}$ & $\Xi$ \\
\hline 126.62 & $\mathbf{3 1 , 8 4 4}$ & $\mathbf{2 5 , 1 5 0}$ & $\mathbf{8 6 5 7}$ & $\mathbf{8 2 0 1 3}$ & $\mathbf{8 6 5 7}$ & $\mathbf{9 4 1 6}$ & $\mathbf{8 2 0 1 3}$ & $\mathbf{1 2 9 0 9}$ & 12758 & $\begin{array}{l}\text { National } \\
\text { Chemical }\end{array}$ & 1 \\
\hline
\end{tabular}


e-ISSN: 2249-4642, p-ISSN: 2454-4671

\begin{tabular}{|c|c|c|c|c|c|c|c|c|c|c|c|}
\hline & & & & & & & & & & Industries & \\
\hline 32.36 & 839 & 2,593 & 2075 & 4050 & 4050 & 2575 & 2140 & 2125 & 2075 & $\begin{array}{l}\text { Iraqi Carpets } \\
\text { \& Furniture }\end{array}$ & 2 \\
\hline 25.94 & 536 , & 2,066 & 1490 & 2937 & 1490 & 1987 & 2084 & 1836 & 2937 & $\begin{array}{l}\text { Baghdad } \\
\text { Packing } \\
\text { Materials } \\
\text { Industry }\end{array}$ & 3 \\
\hline 17.71 & 67,209 & 379,404 & 300580 & 475252 & 475252 & 332500 & 391020 & 300580 & 397670 & $\begin{array}{l}\text { Baghdad Soft } \\
\text { Drinks }\end{array}$ & 4 \\
\hline 93.15 & 10,389 & 11,154 & 1346 & 23417 & 20868 & 23417 & 2173 & 1346 & 7966 & $\begin{array}{l}\text { Readymade } \\
\text { Garments } \\
\text { Production \& } \\
\text { General } \\
\text { Trading } \\
\end{array}$ & 5 \\
\hline 37.66 & 2,181 & 5,791 & 3465 & 8291 & 3465 & 4331 & 4950 & 8291 & 7920 & $\begin{array}{l}\text { Alhlal } \\
\text { Industrial }\end{array}$ & 6 \\
\hline 136.5 & 12,519 & 9,172 & 2520 & 31500 & 4900 & 4140 & 2800 & 31500 & 2520 & Modern sewing & 7 \\
\hline 84.7 & 5,293 & 6,249 & 1089 & 12600 & 10800 & 12600 & 1386 & 1089 & 5373 & $\begin{array}{l}\text { Modern } \\
\text { chemical } \\
\text { products }\end{array}$ & 8 \\
\hline 142.36 & 26,565 & 18,660 & 4514 & 65934 & 4514 & 4693 & 65934 & 6912 & 11250 & $\begin{array}{l}\text { Canadian } \\
\text { Vaccines and } \\
\text { Veterinary } \\
\text { Medicines }\end{array}$ & 9 \\
\hline 12.67 & 632 & 4,993 & 4334 & 5822 & 4464 & 4334 & 4914 & 5434 & 5822 & $\begin{array}{l}\text { AL } \\
\text { MANSOUR } \\
\text { MEDICAL \& } \\
\text { BEAUTY } \\
\text { INDUSTRIES }\end{array}$ & 10 \\
\hline 11.16 & 1,312 & 11,756 & 10319 & 13913 & 11516 & 11516 & 11516 & 13913 & 10319 & Dyes industry & 11 \\
\hline 43.78 & 597 & 1,365 & 945 & 2400 & 1080 & 1050 & 945 & 1350 & 2400 & $\begin{array}{l}\text { Alhlal } \\
\text { Industrial }\end{array}$ & 12 \\
\hline & & 428,097 & 945 & 475252 & 219715 & 230166 & 243299 & 376835 & 530789 & $\begin{array}{l}\text { Average of the } \\
\text { industrial sector }\end{array}$ & \\
\hline 72.2 & 9,844 & 13,635 & 2633 & 23231 & 17966 & 20877 & 2633 & 3469 & 23231 & Sudair hotels & 13 \\
\hline 32.94 & 19,390 & 58,871 & 34754 & 81440 & 34754 & 61383 & 44140 & 72642 & 81440 & $\begin{array}{l}\text { AL } \\
\text { MANSOUR } \\
\text { hotels } \\
\end{array}$ & 14 \\
\hline 16.55 & 1,188 & 7,180 & 5400 & 8000 & 5400 & 8000 & 8000 & 8000 & 6500 & Karbala hotels & 15 \\
\hline 35.99 & 550 & 1,529 & 888 & 2405 & 1428 & 1548 & 888 & 1378 & 2405 & $\begin{array}{l}\text { The tourist city } \\
\text { of Mosul Dam }\end{array}$ & 16 \\
\hline 55.57 & 50,319 & 90,550 & 60750 & 180000 & 75000 & 65000 & 72000 & 180000 & 60750 & Babylon Hotel & 17 \\
\hline
\end{tabular}


e-ISSN: 2249-4642, p-ISSN: 2454-4671

\begin{tabular}{|c|c|c|c|c|c|c|c|c|c|c|c|}
\hline 19.39 & 7,041 & 36,314 & 26721 & 44215 & 32482 & 36440 & 26721 & 41716 & 44215 & Baghdad Hotel & 18 \\
\hline 22.8 & 14,595 & 64,001 & 42018 & 78002 & 56948 & 69285 & 42018 & 73755 & 78002 & Palestine Hotel & 19 \\
\hline 51.43 & 29,686 & 57,721 & 23137 & 1005950 & 40645 & 53151 & 23137 & 105950 & 71079 & $\begin{array}{l}\text { National } \\
\text { Tourism } \\
\text { Investment }\end{array}$ & 20 \\
\hline \multirow[t]{2}{*}{24.04} & 11,269 & 46,877 & 30938 & 556500 & 42000 & 46550 & 30938 & 59250 & 55650 & ISHTAR hotels & 21 \\
\hline & & 41,853 & 888 & 1005950 & 34069 & 40248 & 27830 & 160684 & 102680 & \multicolumn{2}{|c|}{$\begin{array}{l}\text { Average hotel and } \\
\text { tourism sector }\end{array}$} \\
\hline 141.64 & 11,134 & 7,861 & 284 & 27540 & 27540 & 2610 & 284 & 4086 & 4788 & $\begin{array}{l}\text { Iraqi } \\
\text { Agricultural } \\
\text { Production } \\
\text { and Marketing }\end{array}$ & 22 \\
\hline 9.64 & 61, & 640 , & 546 & 702 & 690 & 702 & 633 & 633 & 546 & $\begin{array}{l}\text { National } \\
\text { Agricultural } \\
\text { Production }\end{array}$ & 23 \\
\hline 22.49 & 4,826 & 21,459 & 15180 & 28463 & 15180 & 28463 & 22253 & 21908 & 19492 & $\begin{array}{l}\text { Iraqi Dates } \\
\text { Manufacturing } \\
\text { \& Marketing }\end{array}$ & 24 \\
\hline 25.93 & 667 & 2,574 & 2100 & 3750 & 2415 & 2280 & 2100 & 2325 & 3750 & $\begin{array}{l}\text { Middle East } \\
\text { Lotus Fish } \\
\text { Production }\end{array}$ & 25 \\
\hline 29.24 & 8,684 & 29,701 & 18400 & 39700 & 39700 & 18400 & 23250 & 32505 & 34650 & $\begin{array}{l}\text { Iraqi meat } \\
\text { production and } \\
\text { marketing }\end{array}$ & 26 \\
\hline 29.38 & 653 & 2,224 & 1312 & 3060 & 1312 & 2051 & 2109 & 2592 & 3060 & $\begin{array}{l}\text { Modern } \\
\text { Animal and } \\
\text { Agricultural } \\
\text { Production }\end{array}$ & 27 \\
\hline & & 12,764 & 284 & 39700 & 14472 & 9084 & 8438 & 10674 & 11047 & $\begin{array}{l}\text { Average } \\
\text { agricultural sect }\end{array}$ & \\
\hline 39.25 & 2,132 & 5,433 & 2834 & 8625 & 4400 & 5450 & 5860 & 8625 & 2834 & $\begin{array}{l}\text { Saliva Karkh } \\
\text { tourist city }\end{array}$ & 28 \\
\hline 223.61 & $\mathbf{1 , 8 8 7}$ & 844 & 0 & 4220 & 0 & 0 & 0 & $\mathbf{0}$ & 4220 & $\begin{array}{l}\text { Mosul City } \\
\text { Games }\end{array}$ & 29 \\
\hline 35.41 & 440 & 1,243 & 743 & 1800 & 743 & 847 & 1425 & 1400 & 1800 & $\begin{array}{l}\text { Alnkba } \\
\text { General } \\
\text { Contracting }\end{array}$ & 30 \\
\hline 27.72 & 16,943 & 61,119 & 43282 & 80574 & 43282 & 44544 & 74304 & 80574 & 62892 & $\begin{array}{l}\text { Maamoura } \\
\text { Real Estate } \\
\text { Investments }\end{array}$ & 31 \\
\hline 32.36 & 1,508 & 4,663 & 3132 & 6960 & 3480 & 3132 & 4872 & 4872 & 6960 & $\begin{array}{l}\text { Al Ameen Real } \\
\text { Estate }\end{array}$ & 32 \\
\hline
\end{tabular}


(IJRSSH) 2020, Vol. No. 10, Issue No. I, Jan-Mar

e-ISSN: 2249-4642, p-ISSN: 2454-4671

\begin{tabular}{|c|c|c|c|c|c|c|c|c|c|c|c|}
\hline & & & & & & & & & & Investments & \\
\hline 9.64 & 61 & 640 & 546 & 702 & 690 & 702 & 633 & 633 & 546 & $\begin{array}{l}\text { Baghdad } \\
\text { General } \\
\text { Transport \& } \\
\text { Real Estate }\end{array}$ & 33 \\
\hline & & 12,324 & 0 & 80574 & 13426 & 11322 & 12427 & 23679 & 18518 & \multicolumn{2}{|l|}{$\begin{array}{l}\text { Average service } \\
\text { sector }\end{array}$} \\
\hline & & 31024 & & & 30215 & 26787 & 29093 & 60412 & 46626 & \multicolumn{2}{|c|}{$\begin{array}{l}\text { Arithmetic mean of } \\
\text { all sectors }\end{array}$} \\
\hline & 2.160 & & & & & & & & & \multicolumn{2}{|c|}{$\begin{array}{l}\text { Standard deviation } \\
\text { of all sectors }\end{array}$} \\
\hline 152.72 & & & & & & & & & & \multirow{2}{*}{\multicolumn{2}{|c|}{$\begin{array}{cc}\text { Coefficient of } & \\
& \text { var } \\
& \text { iati } \\
& \text { on } \\
& \text { for } \\
& \text { all } \\
\text { sec } & \\
\text { tor } & \text { s }\end{array}$}} \\
\hline & & & & & & & & & & & \\
\hline
\end{tabular}

4-5 - Relationship between credit policies and market value: Relationship between Credit Policies and Market Value

From Table (8) we note that there is a great disparity between the policies of commercial credit granted and earned, as some companies adopted the conservative policy of the grantee and earned and some others used the moderate policy as well as the grantee and earned and some third used the conservative trade credit granted and earned and some others used a combination of those different policies In order to reach the increase in the market value, from Table (8) we see that most of the research samples regardless of the type of sector as well as the years that the companies that obtain a credit earned greater than the grant of commercial credit granted or be equal between the grantee and the acquired six Being the best in terms of the market value in most cases, the research sample is seen, and this does not prevent the existence of adverse cases, but this has been reached through this combination of commercial credit policies granted and earned and table (7) shows the symbols used.

Table (7) Symbols used in the research

\begin{tabular}{|l|l|l|}
\hline Name & Symbols & $\Xi$ \\
\hline High efficiency or value & $R$ & 1 \\
\hline Efficiency or medium value & W & 2 \\
\hline Low efficiency or value & $N$ & 3 \\
\hline Risky policy & $J$ & 4 \\
\hline Moderate policy & A & 5 \\
\hline Conservative policy & $M$ & 6 \\
\hline
\end{tabular}


Table (8) Credit Policies Granted, Earned, and Market Value for Years and Sample Companies

\begin{tabular}{|c|c|c|c|c|c|c|}
\hline 2017 & 2016 & 2015 & 2014 & 2013 & The Company's name & ت \\
\hline A-J-N & A-J-N & $\mathbf{A}-\mathbf{A}-\mathbf{N}$ & M-J-N & A-J-N & $\begin{array}{l}\text { National Chemical } \\
\text { Industries }\end{array}$ & 1 \\
\hline $\mathbf{M}-\mathbf{A}-\mathbf{N}$ & M-J-N & M-J-N & M-J-N & M-J-N & $\begin{array}{l}\text { Iraqi Carpets \& } \\
\text { Furniture }\end{array}$ & 2 \\
\hline $\mathbf{A}-\mathbf{A}-\mathbf{N}$ & $\mathbf{M}-\mathbf{A}-\mathbf{N}$ & $\mathbf{M}-\mathbf{A}-\mathbf{N}$ & $\mathbf{M}-\mathbf{A}-\mathbf{N}$ & $\mathbf{M}-\mathbf{A}-\mathbf{N}$ & $\begin{array}{l}\text { Baghdad Packing } \\
\text { Materials Industry }\end{array}$ & 3 \\
\hline M-M-R & M-A-R & M-A-R & M-M-R & A-A-R & Baghdad Soft Drinks & 4 \\
\hline $\mathbf{A}-\mathbf{A}-\mathbf{R}$ & A-J-R & $\mathbf{J}-\mathbf{J}-\mathbf{N}$ & A-J-N & A-J-N & $\begin{array}{l}\text { Readymade Garments } \\
\text { Production \& General } \\
\text { Trading }\end{array}$ & 5 \\
\hline A-J-N & A-J-N & A-J-N & A-J-N & A-J-N & Alhlal Industrial & 6 \\
\hline $\mathbf{A}-\mathbf{A}-\mathbf{N}$ & $\mathbf{A}-\mathbf{A}-\mathbf{N}$ & $\mathbf{A}-\mathbf{A}-\mathbf{N}$ & $\mathbf{M}-\mathbf{A}-\mathbf{N}$ & M-A-N & Modern sewing & 7 \\
\hline $\mathbf{M}-\mathbf{A}-\mathbf{N}$ & A-J-N & M-J-N & M-J-N & M-M-A & $\begin{array}{l}\text { Modern chemical } \\
\text { products }\end{array}$ & 8 \\
\hline A-M-N & M-M-N & M-A-N & $\mathbf{M}-\mathbf{A}-\mathbf{N}$ & M-M-N & $\begin{array}{l}\text { Canadian Vaccines and } \\
\text { Veterinary Medicines }\end{array}$ & 9 \\
\hline M-J-N & A-J-N & A-J-N & A-J-N & J-A-N & $\begin{array}{l}\text { AL MANSOUR } \\
\text { MEDICAL \& BEAUTY } \\
\text { INDUSTRIES }\end{array}$ & 10 \\
\hline M-M-N & $\mathbf{M}-\mathbf{A}-\mathbf{N}$ & M-M-N & $\mathbf{M}-\mathbf{A}-\mathbf{N}$ & A-M-N & Dyes industry & 11 \\
\hline M-A-N & $\mathbf{M}-\mathbf{A}-\mathbf{N}$ & A-A-N & $\mathbf{M}-\mathbf{A}-\mathbf{N}$ & $\mathbf{A}-\mathbf{A}-\mathbf{N}$ & Alhlal Industrial & 12 \\
\hline $\mathbf{J}-\mathbf{J}-\mathbf{N}$ & A-J-N & $\mathbf{J}-\mathbf{J}-\mathbf{N}$ & $\mathbf{J}-\mathbf{A}-\mathbf{N}$ & $\mathbf{J}-\mathbf{J}-\mathbf{N}$ & Sudair hotels & 13 \\
\hline A-J-R & A-J-R & A-J-R & J-J-R & J-J-R & AL MANSOUR hotels & 14 \\
\hline $\mathbf{M}-\mathbf{A}-\mathbf{N}$ & M-M-N & $\mathbf{M}-\mathbf{A}-\mathbf{N}$ & M-J-N & M-M-N & Karbala hotels & 15 \\
\hline J-A-R & $\mathbf{J}-\mathbf{J}-\mathbf{N}$ & $\mathbf{J}-\mathbf{J}-\mathbf{N}$ & $\mathbf{J}-\mathbf{J}-\mathbf{N}$ & A-J-N & $\begin{array}{l}\text { The tourist city of Mosul } \\
\text { Dam }\end{array}$ & 16 \\
\hline A-J-R & MJ-R & A-J-R & A-J-R & M-J-R & Babylon Hotel & 17 \\
\hline M-J-R & M-A-R & M-J-R & M-J-R & M-A-R & Baghdad Hotel & 18 \\
\hline A-J-R & A-J-R & A-J-R & A-J-R & A-J-R & Palestine Hotel & 19 \\
\hline A-M-R & M-A-R & M-A-R & M-A-R & A-A-R & $\begin{array}{l}\text { National Tourism } \\
\text { Investment }\end{array}$ & 20 \\
\hline A-J-R & J-M-R & A-J-R & A-J-R & A-M-R & ISHTAR hotels & 21 \\
\hline $\mathbf{J}-\mathbf{A}-\mathbf{R}$ & $\mathbf{M}-\mathbf{A}-\mathbf{N}$ & A-A-N & $\mathbf{M}-\mathbf{A}-\mathbf{N}$ & $\mathbf{M}-\mathbf{A}-\mathbf{N}$ & $\begin{array}{l}\text { Iraqi Agricultural } \\
\text { Production and }\end{array}$ & 22 \\
\hline
\end{tabular}




\begin{tabular}{|c|c|c|c|c|c|c|}
\hline & & & & & Marketing & \\
\hline $\mathbf{J}-\mathbf{J}-\mathbf{N}$ & A-J-N & M-A-N & M-J-N & A-A-N & $\begin{array}{l}\text { National Agricultural } \\
\text { Production }\end{array}$ & 23 \\
\hline $\mathbf{J}-\mathbf{J}-\mathbf{N}$ & A-J-N & $\mathbf{J}-\mathbf{J}-\mathbf{N}$ & A-A-N & A-A-N & $\begin{array}{l}\text { Iraqi Dates } \\
\text { Manufacturing \& } \\
\text { Marketing }\end{array}$ & 24 \\
\hline M-J-N & M-A-N & M-A-N & M-J-N & M-A-N & $\begin{array}{l}\text { Middle East Lotus Fish } \\
\text { Production }\end{array}$ & 25 \\
\hline J-J-R & J-J-R & J-J-R & J-J-R & J-J-R & $\begin{array}{l}\text { Iraqi meat production } \\
\text { and marketing }\end{array}$ & 26 \\
\hline A-J-N & A-A-N & $\mathbf{A}-\mathbf{A}-\mathbf{N}$ & $\mathbf{A}-\mathbf{A}-\mathbf{N}$ & A-A-N & $\begin{array}{l}\text { Modern Animal and } \\
\text { Agricultural Production }\end{array}$ & 27 \\
\hline M-A-N & A-M-N & M-M-N & A-J-N & M-M-N & Saliva Karkh tourist city & 28 \\
\hline A-J-N & A-J-N & A-J-N & A-J-N & A-J-N & Mosul City Games & 29 \\
\hline A-A-R & M-M-R & A-A-R & A-A-R & M-M-R & $\begin{array}{l}\text { Alnkba General } \\
\text { Contracting }\end{array}$ & 30 \\
\hline A-A-N & A-A-N & A-A-N & A-A-N & A-J-N & $\begin{array}{l}\text { Maamoura Real Estate } \\
\text { Investments }\end{array}$ & 31 \\
\hline A-J-N & M-A-N & A-J-N & A-A-N & A-A-N & $\begin{array}{l}\text { Al Ameen Real Estate } \\
\text { Investments }\end{array}$ & 32 \\
\hline A-A-N & A-M-N & J-A-N & J-A-N & M-A-N & $\begin{array}{l}\text { Baghdad General } \\
\text { Transport \& Real Estate }\end{array}$ & 33 \\
\hline
\end{tabular}

\section{4-6-Relationship between Credit Policies and Efficiency and Market Value}

Table (9) shows the efficiency of the credit granted and earned and the market value of the companies. Where the views varied between high and medium and low level, the earned credit is the efficiency of short average repayment period and the high turnover of commercial credit earned and vice versa and result in this research three levels of efficiency (high, medium, low) as those Companies that have varied between high, low and medium efficiency or a combination of those policies for granted and earned credit. As a result of the leniency of the sales department with customers in granting a longer period to customers for the purpose of repayment, this thing in the present is an effective promotion in obtaining a larger market share, while the high efficiency in the credit earned improves the perceived image of the government $\mathrm{P}$ and public opinion as well as it is free to promote the company's products to achieve this, a greater profit and consequently leads to a rise in the market value of the company

Table (9) Credit Efficiency Granted and Earned and Market Value of Companies

\begin{tabular}{|c|c|c|c|c|l|c|}
\hline 2017 & 2016 & 2015 & 2014 & 2013 & \multicolumn{1}{|l|}{ The Company's name } & \multirow{2}{|c|}{} \\
\hline N-N-N & N-N-N & N-N-N & R-N-N & N-N-N & $\begin{array}{l}\text { National Chemical } \\
\text { Industries }\end{array}$ & 1 \\
\hline N-W-N & N-W-N & N-N-N & N-W-N & N-W-N & $\begin{array}{l}\text { Iraqi Carpets \& } \\
\text { Furniture }\end{array}$ & 2 \\
\hline N-W-N & N-W-N & W-W-N & N-W-N & N-W-N & $\begin{array}{l}\text { Baghdad Packing } \\
\text { Materials Industry }\end{array}$ & 3 \\
\hline
\end{tabular}




\begin{tabular}{|c|c|c|c|c|c|c|}
\hline W-R-R & W-R-R & W-R-R & W-R-R & W-R-R & Baghdad Soft Drinks & 4 \\
\hline N-N-R & N-N-R & $\mathbf{N}-\mathbf{N}-\mathbf{N}$ & W-N-N & $\mathbf{N}-\mathbf{N}-\mathbf{N}$ & $\begin{array}{l}\text { Readymade Garments } \\
\text { Production \& General } \\
\text { Trading }\end{array}$ & 5 \\
\hline $\mathbf{N}-\mathbf{N}-\mathbf{N}$ & R-N-N & $\mathbf{N}-\mathbf{N}-\mathbf{N}$ & $\mathbf{N}-\mathbf{N}-\mathbf{N}$ & $\mathbf{N}-\mathbf{N}-\mathbf{N}$ & Alhlal Industrial & 6 \\
\hline $\mathbf{N}-\mathbf{N}-\mathbf{N}$ & N-N-N & $\mathbf{N}-\mathbf{W}-\mathbf{N}$ & R-N-N & N-N-N & Modern sewing & 7 \\
\hline W-N-N & $\mathbf{N}-\mathbf{N}-\mathbf{N}$ & R-N-N & $\mathbf{W}-\mathbf{N}-\mathbf{N}$ & N-N-N & $\begin{array}{l}\text { Modern chemical } \\
\text { products }\end{array}$ & 8 \\
\hline N-N-N & N-N-N & $\mathbf{N}-\mathbf{N}-\mathbf{N}$ & $\mathbf{N}-\mathrm{N}-\mathbf{N}$ & $\mathbf{N}-\mathbf{N}-\mathbf{N}$ & $\begin{array}{l}\text { Canadian Vaccines and } \\
\text { Veterinary Medicines }\end{array}$ & 9 \\
\hline N-W-N & $\mathbf{N}-\mathbf{W}-\mathbf{N}$ & $\mathbf{N}-\mathbf{N}-\mathbf{N}$ & $\mathbf{N}-\mathbf{N}-\mathbf{N}$ & N-W-N & $\begin{array}{l}\text { AL MANSOUR } \\
\text { MEDICAL \& BEAUTY } \\
\text { INDUSTRIES }\end{array}$ & 10 \\
\hline $\mathbf{N}-\mathbf{N}-\mathbf{N}$ & $\mathbf{N}-\mathbf{N}-\mathbf{N}$ & $\mathbf{N}-\mathbf{N}-\mathbf{N}$ & $\mathbf{N}-\mathbf{N}-\mathbf{N}$ & $\mathbf{N}-\mathbf{N}-\mathbf{N}$ & Dyes industry & 11 \\
\hline N-N-N & N-N-N & $\mathbf{N}-\mathbf{N}-\mathbf{N}$ & W-N-N & N-W-N & Alhlal Industrial & 12 \\
\hline $\mathbf{N}-\mathbf{N}-\mathbf{N}$ & R-N-N & R-N-N & $\mathbf{N}-\mathbf{N}-\mathbf{N}$ & N-W-N & Sudair hotels & 13 \\
\hline N-N-R & N-N-R & R-N-R & N-N-R & N-N-R & AL MANSOUR hotels & 14 \\
\hline $\mathbf{N}-\mathbf{N}-\mathbf{N}$ & N-N-N & R-R-N & R-N-N & W-N-N & Karbala hotels & 15 \\
\hline N-N-N & N-N-N & $\mathbf{N}-\mathbf{N}-\mathbf{N}$ & N-N-N & N-N-N & $\begin{array}{l}\text { The tourist city of Mosul } \\
\text { Dam }\end{array}$ & 16 \\
\hline R-N-R & R-N-R & R-N-R & R-N-R & R-N-R & Babylon Hotel & 17 \\
\hline N-N-R & N-N-R & W-W-R & N-N-R & N-N-R & Baghdad Hotel & 18 \\
\hline N-N-R & W-N-R & N-N-R & R-N-R & W-N-R & Palestine Hotel & 19 \\
\hline N-N-R & N-W-R & N-N-R & W-N-R & W-N-R & $\begin{array}{l}\text { National Tourism } \\
\text { Investment }\end{array}$ & 20 \\
\hline N-N-R & N-N-R & N-R-R & N-W-R & N-W-R & ISHTAR hotels & 21 \\
\hline W-N-R & W-N-N & $\mathbf{N}-\mathbf{N}-\mathbf{N}$ & N-N-N & R-N-N & $\begin{array}{l}\text { Iraqi Agricultural } \\
\text { Production and } \\
\text { Marketing } \\
\end{array}$ & 22 \\
\hline N-W-N & N-W-N & N-N-N & N-W-N & N-N-N & $\begin{array}{l}\text { National Agricultural } \\
\text { Production }\end{array}$ & 23 \\
\hline N-N-N & N-N-N & N-N-N & N-N-N & N-W-N & $\begin{array}{l}\text { Iraqi Dates } \\
\text { Manufacturing \& } \\
\text { Marketing }\end{array}$ & 24 \\
\hline N-N-N & N-N-N & N-N-N & N-N-N & N-N-N & $\begin{array}{l}\text { Middle East Lotus Fish } \\
\text { Production }\end{array}$ & 25 \\
\hline
\end{tabular}




\begin{tabular}{|c|c|c|c|c||l|l|}
\hline N-W-R & N-N-R & N-N-R & N-N-R & N-W-R & $\begin{array}{l}\text { Iraqi meat production } \\
\text { and marketing }\end{array}$ & 26 \\
\hline N-N-N & R-N-N & N-N-N & N-N-N & N-N-N & $\begin{array}{l}\text { Modern Animal and } \\
\text { Agricultural Production }\end{array}$ & 27 \\
\hline W-N-N & R-N-N & R-N-N & N-N-N & W-N-N & Saliva Karkh tourist city & 28 \\
\hline N-N-N & N-N-N & N-N-N & N-N-N & R-N-N & Mosul City Games & 29 \\
\hline N-N-R & R-N-R & N-N-R & N-N-R & N-N-R & $\begin{array}{l}\text { Alnkba General } \\
\text { Contracting }\end{array}$ & 30 \\
\hline W-N-N & N-N-R & N-N-N & N-N-N & N-N-N & $\begin{array}{l}\text { Maamoura Real Estate } \\
\text { Investments }\end{array}$ & 31 \\
\hline N-N-N & N-N-N & R-N-N & N-N-N & N-N-N & $\begin{array}{l}\text { Al Ameen Real Estate } \\
\text { Investments }\end{array}$ & 32 \\
\hline N-N-N & N-N-N & N-N-N & N-N-N & N-N-N & $\begin{array}{l}\text { Baghdad General } \\
\text { Transport \& Real Estate }\end{array}$ & 33 \\
\hline
\end{tabular}

4-7 The relationship between the policies and the efficiency of commercial credit granted and earned

Through Table (10), we will summarize the relationship between trade credit granted and earned and what is the best policy that achieves a high level of efficiency? The table shows that the best policy according to the efficiency standards is the one that achieves the highest efficiency, which is both the policy (risk-conservative, conservative-moderate) while the worst policy in achieving the lowest efficiency is the policy (moderate - moderate).

Table (10) Type of credit policies granted and earned and efficiency levels

\begin{tabular}{|c|c|c|c|c|c|}
\hline Level of efficiency & percentage & number & Credit earned & Credit granted & \\
\hline 2.6 & 0.06 & 11 & Conservative & Conservative & 1 \\
\hline 2.3 & 0.01 & 2 & Conservative & Moderate & 2 \\
\hline 3.3 & 0.01 & 2 & Conservative & Risky & 3 \\
\hline 3.1 & 0.24 & 38 & Moderate & Conservative & 4 \\
\hline 2.1 & 0.13 & 23 & Moderate & Moderate & 5 \\
\hline 2.2 & 0.06 & 10 & Moderate & Risky & 6 \\
\hline 3.1 & 0.13 & 22 & Risky & Conservative & 7 \\
\hline 2.6 & 0.26 & 42 & Risky & Moderate & 8 \\
\hline 2.3 & 0.10 & 15 & Risky & Risky & 9 \\
\hline & 1.00 & 165 & $=$ & $=$ & Total \\
\hline
\end{tabular}

\section{CONCLUSIONS}

The results showed that the long-term buying and selling operations carried out by the business companies (research sample) are not based on clear policies and this is reflected negatively on the profitability of companies and their market value. Some results confirm the impact of commercial credit policies (conservative, moderate, and risky). Most businesses (research sample) and across all sectors of the research sample adopted a moderate policy in the management of credit granted to other ratios, although there is a variation in the extent Conformity between the policy followed and the level of market value of the entity. The results showed that the majority of businesses (research sample) and across 
all sectors of the research sample adopted the policy of risk management in the earned credit towards other ratios, although there is a discrepancy in the extent of convergence between the policy followed and the level of market value of the facility. The combination adopted by most research sample companies is to obtain credit earned more than granted credit.

\section{SOURCES}

1- $\quad \mathrm{Wu}$,Wanlin, \&Michaildis, Alexandros, Impacts of Credit Rating Announcements on Share Price in the NASDAQ Market and the Role Of the Credit Rating Agencies, Lund University School of Economics and Management, 2014

2- $\quad$ Ehrhardt, Michael C. Brigham Eugene F, Financial Management: Theory and Practice, South-Western, a part of Cengage Learning $13^{\text {th }}$ ed, 2012

3- Chou ,Jian-Hsin, Mei-Ching Yang \&Tsung-Te Lin, (2011), "An Empirical Analysis of the Effect of Credit Rating on Trade Credit" , 2011 International Conference on Financial Management and Economics IPEDR vol.11, IACSIT Press, Singapore .

4- Olusola ,Ojenike Joseph, (2012) Use of Trade Credit in Nigeria: A Panel Econometric Approach , Research Journal of Finance and Accounting, Vol 3, No 2 .

5- $\quad$ Pike . Richard \& Bill Neale 2006,"Corporate Finance And Investment Decisions \& Strategies" , $5^{\text {th }}$. ed. , Pearson Hall, Spain., .

6- Beck, T. \&Demirguc-Kunt, A. (2006). Small and medium0size enterprises: Access to finance as a growth constraint. Journal of Banking \& Finance 30 2931-2943.

7- $\quad$ DarushYazdanfar Peter Öhman , (2016),"The impact of trade credit use on firm profitability: empirical evidence from Sweden", Journal of Advances in Management Research, Vol. 13 Iss 2 pp. 1-16

8- Al Dohaiman, Mohammed Saleh ,(2013), Explaining the Determinants of Trade Credit: An Empirical Study in the Case of Saudi Arabian's unlisted Firms , Research Journal of Finance and Accounting Vol.4, No.17.

9- Niskanen, J., Niskanen, M. (2000). Accounts Receivable and Accounts Payable in Large Finnish Firms Balance Sheets: What Determines Their Levels? The Finnish Journal of Business Economics, 489-503.

10- $\quad$ FISMAN , RAYMOND and INESSA LOVE (2011),"Trade Credit, Financial Intermediary Development, and Industry Growth" , THE JOURNAL OF FINANCE, VOL. LVIII, NO. 1 , FEB.

11- García-Teruel, P. J., \&Martínez-Solano, P. (2010). A dynamic perspective on the determinants of accounts payable. Rev Quant Finan ,Acc, 34, 439-457. doi: 1007/s11156-009-0124-0

12- Kwenda ,Farai,(2014) ,"Trade Credit In Zimbabwe's Economic Recovery" Mediterranean Journal of Social Sciences, MCSER Publishing, Rome-Italy ,Vol 5 No 2.

13- Solaa, Cristina Martı'nez\&Teruelb, Pedro J. Garcıa' Solanoa , Pedro Martı'nez- (2018) Trade credit policy and firm value , doi: 10.1111/j.1467-629X.2012.0048

14- Malik, M. F., Qureshi, M. U. and Azeem, M, 2012. Determination of Share Price: Evidence from Karachi Stock 15- NTHENGE, MWANGANGI JAMES , (2013) , RELATIONSHIP BETWEEN TRADE CREDIT AND VALUE OF FIRMS LISTED AT THE NAIROBI SECURITIES EXCHANGE, SCHOOL OF BUSINESS UNIVERSITY OF NAIROBI, THE DEGREE OF MASTER . 